\title{
Qualidade do sono dos profissionais da saúde que trabalham em regime de plantão noturno: revisão sistemática da literatura
}

\author{
Sleep quality of healthcare professionals working in the night duty regime: systematic literature \\ review
}

Calidad del sueño de los profesionales de la salud que trabajan en el régimen de servicio nocturno: revisión sistemática de la literatura

\section{Resumo}

Objetivo: Elaborar uma revisão sistemática de literatura analisando a qualidade do sono de profissionais da saúde em regime de plantão noturno. Metodologia: Realizou-se a revisão sistemática de literatura, com pesquisa nas bases de dados eletrônicas PubMed, Biblioteca Virtual em Saúde, SCIELO. Foram incluídos estudos que abordaram a qualidade do sono de profissionais da saúde que trabalham em regime de plantão noturno; publicados a partir de 2016 a 2021 e disponibilizados nas bases de dados, nos idiomas inglês e português; com participantes de idade superior a 18 anos. Foram excluídos artigos de revisão; artigos cujos participantes não eram profissionais da saúde e os que possuíam diagnóstico prévio de distúrbios do sono. Resultados: Do total de 3.540 trabalhos encontrados nas bases de dados eletrônicas, 26 eram referentes a Scielo, 1.523 foram encontrados na PubMed e 1.991 na BVS. Foram excluídos 3.464 artigos e, a partir dos 76 estudos selecionados, realizou-se a busca manual, totalizando 21 artigos. Discussão: $\mathrm{O}$ excesso de atividades e altas jornadas de trabalho, além das alterações do sono podem contribuir em problemáticas que afetem a vida social, psicológica e fisiológica dos trabalhadores da saúde em regime de plantão noturno, afetando negativamente não só os profissionais, mas também o ambiente de trabalho, a equipe e os próprios pacientes. Conclusão: Os profissionais que mais sofrem conflitos diários em suas jornadas de trabalho são os que os realizam em regime de plantão hospitalar noturno, porém podem obter alívio das consequências a partir de elaboração de alternativas de escala. Palavras-chave: Sono; Transtorno do sono-vigília; Jornada de trabalho em turnos.

\footnotetext{
Abstract

Objective: Develop a systematic literature review analysing the sleep quality of health professionals on night shift. Method: A systematic literature review was carried out, with search in the electronicdatabases PubMed, Virtual Health Library, SCIELO. Studies that addressed the quality of sleep of health professionals who work on a night shift were included; published from 2016 to 2021 and made available in the databases, in English and Portuguese; whose participants were over 18 years of age. Review articles were excluded; articles whose participants were not health
} 
professionals and those who had a previous diagnosis of sleep disorders. Results: Of the total of 3,540 works found in the electronic databases, 26 were related to Scielo, 1,523 were found in PubMed, and 1,991 in the VHL (Virtual Health Library). 3,464 articles were excluded, from the 76 selected studies, and a manual search was performed, totaling 21 articles. Discussion: Excessive activities andlong working hours, in addition to sleep disorders, can directly contribute to the problems that affect the social, psychological, and physiological life of healthworkers on night shifts, negatively affecting not only the professionals but also the work environment, the team and the patients themselves. Conclusion: The professionals who suffer the most daily conflicts in their workdays are those who perform them on a night shift in hospital, but they can obtain relief from the consequences through the elaboration of alternative scales.

Keywords: Sleep; Sleep wake disorders; Shift work schedule.

\section{Resumen}

Objetivo: Desarrollar una revisión sistemática de la literatura analizando la calidad del sueño de los profesionales de la salud en los turno nocturnos. Metodología: Se realizó una revisión bibliográfica sistemática, con búsqueda en las bases de datos electrónicas PubMed, Biblioteca Virtual en Salud, SCIELO. Se incluyeron los estudios que abordaron la calidad del sueño de los profesionales de la salud que trabajan en turno de noche; publicado de 2016 a 2021 y disponible en las bases de datos, en inglés y portugués; con participantes mayores de 18 años. Se excluyeron los artículos de revisión; artículos cuyos participantes no eran profesionales de la salud y que tenían un diagnóstico previo de trastornos del sueño. Resultados: Del total de 3.540 trabajos encontrados en bases de datos electrónicas, 26 estaban relacionados con Scielo, 1.523 se encontraron en PubMed y 1.991 en la BVS. Se excluyeron 3.464 artículos y, de los 76 estudios seleccionados, se realizó una búsqueda manual, totalizando 21 artículos. Discusión: Las actividades excesivas y las largas jornadas laborales, además de los trastornos del sueño pueden contribuir a problemas que afectan la vida social, psicológica y fisiológica de los trabajadores de la salud en los turnos nocturnos, afectando negativamente no solo a los profesionales, sino también al ambiente de trabajo, al equipo y a la propios pacientes. Conclusión: Los profesionales que sufren más conflictos cotidianos en su jornada laboral son los que los realizan en turno de noche en el hospital, pero pueden obtener alivio de las consecuencias mediante la elaboración de escalas alternativas.

Palabras clave: Sueño; Trastornos del sueño-vigilia; Horario de trabajo por turnos.

\section{Introdução}

O sono tem sido definido como um estado fisiológico complexo, que requer uma integração cerebral completa durante a qual ocorrem alterações dos processos fisiológicos e comportamentais (Basner et al., 2019). Assim, é um processo biológico natural do organismo, essencial para reparação e manutenção do equilíbrio biopsicossocial dos seres vivos, posto que, biologicamente, o ser humano não foi desenvolvido na cadeia natural para práticas noturnas, tendo o seu padrão circadiano alterado para que em face das necessidades de consumo e trabalho, se enquadrassem nesse novo modo de vida (Barger et al., 2019).

Sabe-se que a privação do sono aumenta os riscos de acidentes de trabalho, sintomas neurocomportamentais e redução da qualidade de vida. Dessa forma o trabalho em turnos acarreta problemas relacionados ao sono, como privação do sono, má qualidade do sono e sonolência diurna (Francis et al., 2019). Estudos têm demonstrado que os plantões noturnos e a privação de sono estão entre as principais causas de erros médicos, como erros de medicação, uso indevido de dispositivos médicos, ferimentos por agulhas, erros cirúrgicos e quedas de pacientes (Arzalier-Daret et al., 2018).

Nesse contexto os médicos que trabalham no ambiente de Serviços Médicos de Emergência são trabalhadores por turnos que costumam trabalhar 12 ou 24 horas consecutivas com períodos de maior estresse e atendimento clínico de emergência separados por períodos variáveis de descanso. O trabalho em turnos, ou seja, o trabalho realizado fora do horário normal de luz do dia, tem sido associado à privação do sono, fadiga e resultados negativos de segurança para pacientes e médicos em vários ambientes de saúde. Metade dos médicos referem dormir 6 horas por dia, mais da metade relata má qualidade do sono e fadiga, e metade relata má recuperação entre os turnos programados (Phoy \& Keogh, 2019). Sendo assim muitos médicos de emergência terão tomado medidas para minimizar sua privação pessoal de sono por meio do uso de sonífero farmacológico (Basner et al., 2019).

A perda de sono e o trabalho noturno estão associados à redução do estado de alerta e do desempenho cognitivo e representam um desafio para os profissionais da saúde que trabalham em turnos (Liu et al,. 2019). As programações destes 
profissionais geralmente incluem turnos noturnos e rotativos, horários de início pela manhã ou noite e horas de trabalho complementares (Nishida et al., 2017). No entanto, a maioria dos profissionais da saúde estão sujeitos a um considerável estresse psicológico, fadiga e privação de sono, o que tem um grande impacto em todos os aspectos de suas vidas, incluindo efeitos prejudiciais no aprendizado e no desempenho clínico (Gómez-García et al., 2016).

Os profissionais de saúde que trabalham em regime de plantão noturno, compõem em sua maioria trabalhadores que possuem elevados fatores de risco cardiometabólicos, incluindo níveis mais elevados de triglicerídeos e níveis mais baixos de lipoproteína de alta densidade (HDL) - colesterol. Estes profissionais também compreendem uma maior incidência em adquirir doenças crônicas do que a população trabalhadora em geral, incluindo síndrome metabólica e obesidade. O trabalho noturno também está relacionado a um risco aumentado de diabetes, pressão arterial, câncer de mama e doenças cardíacas (Abbott et al., 2019)

Ao considerar que o processo de trabalho noturno pode acarretar problemas fisiológicos, sociais e que isso pode contribuir para situações de imprudência e negligência que afetam plantões trabalhados, surgiu o interesse dos autores em realizar uma revisão sistemática de literatura analisando a qualidade do sono de profissionais da saúde em regime de plantão noturno. Desse modo, obteve-se a seguinte pergunta norteadora: Qual a qualidade do sono de profissionais da saúde que trabalham nos regimes de plantão noturno e as possíveis consequências dessa prática?

\section{Metodologia}

Realizou-se uma análise bibliográfica descritiva, tipo Revisão Sistemática de Literatura, que possibilitou a compreensão e delimitação do tema proposto e da pergunta norteadora mediante a utilização da estratégia PICO, que representa um acrônimo para Paciente, Intervenção, Comparação e "Outcomes" (desfecho), utilizado pela Medicina Baseada em Evidência. Diante disso, elaborou-se a seguinte questão norteadora: Qual a qualidade do sono de profissionais da saúde que trabalham nos regimes de plantão noturno e as possíveis consequências dessa prática?

Foi realizada a revisão sistemática de literatura, no período de junho de 2020, com pesquisa nas bases de dados eletrônicas PubMed, Biblioteca Virtual em Saúde, SCIELO. Como descritores, utilizou-se os termos dos Descritores em Ciências da Saúde (DeCS) correspondentes a "Sleep" AND "Sleep disorders" AND "shift work", obtendo-se o detalhamento de busca: ((Sleep) AND (Sleep disorders)) AND (shift work). Adicionalmente, realizou-se a busca manual nas referências dos artigos selecionados.

Foram incluídos estudos que abordaram a Qualidade do sono de profissionais da saúde que trabalham em regime de plantão noturno; publicados a partir de 2016 a 2021 e disponibilizados nas bases de dados, visando contemplar os trabalhos mais atualizados, abrangendo o período de 5 anos; nos idiomas inglês e português; cujos participantes apresentavam idade superior a 18 anos. Foram excluídos artigos de revisão; pesquisas cujos participantes não eram profissionais da saúde e os que possuíam diagnóstico prévio de distúrbios do sono.

Os trabalhos foram identificados por dois grupos de três autores que, de forma independente, avaliaram seus títulos e resumos, triando-os conforme os critérios de inclusão e exclusão. Os trabalhos sem resumo, ou com resumo insuficiente para avaliação, ou que não apresentavam o texto disponível na íntegra, não excluídos pelo título, foram também triados para leitura do texto completo. Cada autor avaliou separadamente os textos completos juntamente com os dados disponíveis, para inclusão nesta revisão sistemática. A busca manual seguiu o mesmo princípio de seleção. Nos casos de divergências, um terceiro autor foi consultado.

Foram coletados os seguintes dados dos artigos selecionados: título; autor(es); ano de publicação; país de origem; revista científica de publicação; idioma; palavras-chave; objetivo(s); desenho de estudo; método; período de realização; critérios de inclusão e exclusão; tamanho da amostra; idade dos participantes; sexo dos participantes; escala de trabalho; área de atuação 
profissional; características clínicas dos trabalhadores de turno; comorbidades; distúrbios alimentares associados; outros resultados da pesquisa; e conclusões.

Para a análise dos artigos foram considerados para caracterização a autoria, o ano de publicação, título, bibliotecas virtuais, objetivo, método e níveis de evidência. Esses estudos foram subdivididos em estudos de forte evidência (metanálise de múltiplos estudos controlados - Nível 1; estudo individual com delineamento experimental - Nível 2); de moderada evidência (pesquisas com delineamento quase-experimental como estudo sem randomização com grupo único após ou antes de teste, séries temporais ou caso controle - Nível 3; estudo com delineamento não experimental como estudos de caso - Nível 4; relatórios de casos ou dado obtido de forma sistemática, de qualidade verificável ou dados de avaliação de programas - Nível 5); de baixa evidência (opiniões de autoridades baseada na competência clínica ou opinião de comitês de especialistas, incluindo interpretações de informações não baseadas em pesquisas - Nível 6). E, por fim, foi realizado a interpretação e discussão dos resultados obtendo a síntese dos conhecimentos apresentados.

\section{Resultados}

Do total de 3.540 trabalhos encontrados nas bases de dados eletrônicas, 26 eram referentes a Scielo, 1.523 foram encontrados na PubMed e 1.991 na BVS (Biblioteca Virtual em Saúde). Foram excluídos 3.464 artigos e, a partir dos 76 estudos selecionados, realizou-se a busca manual, totalizando 21 artigos, todos elegíveis para a presente revisão sistemática (Figura 1). Permaneceram 21 estudos que atendiam as condições para inclusão na presente revisão sistemática, distribuídos entre: 1 da Scielo, 7 da PubMed e 13 da BVS (Figura 2).

Barger et al. (2019) compararam as horas de trabalho do médico residente e o sono em um ensaio clínico multicêntrico cruzado randomizado agrupado que randomizou os médicos residentes a uma Escala de Trabalho de Duração Estendida (Extended Duration Work Roster) com turnos de duração estendida ( $\geq 24$ horas) ou uma Escala de Trabalho de Ciclo Rápido (Rapidly Cycling Work Roster), em que a duração dos turnos programados foi limitada a 16 ou menos horas consecutivas.

Blackwell et al. (2019) realizaram um estudo multicêntrico não cego em 6 UTI Pediátricas de centros médicos acadêmicos dos Estados Unidos. O objetivo principal da pesquisa era comparar a segurança do paciente entre a Escala de Trabalho de Duração Estendida (EDWR), que incluía turnos $\geq 24$ h, e uma Escala de Trabalho de Ciclo Rápido (RCWR), onde os turnos eram limitados a um máximo de 16 h, também utilizada no estudo de Barger, et al. (2019) cuja as informações sobre erros médicos potenciais foram coletadas e usadas para classificação por revisores médicos treinados centralmente, que desconheciam o braço do estudo, objetivando avaliar a relação do braço do estudo com a duração do sono do médico residente, horas de trabalho e desempenho neurocomportamental. 
Figura 1. Fluxograma de identificação, triagem, elegibilidade e inclusão de estudos na revisão sistemática.

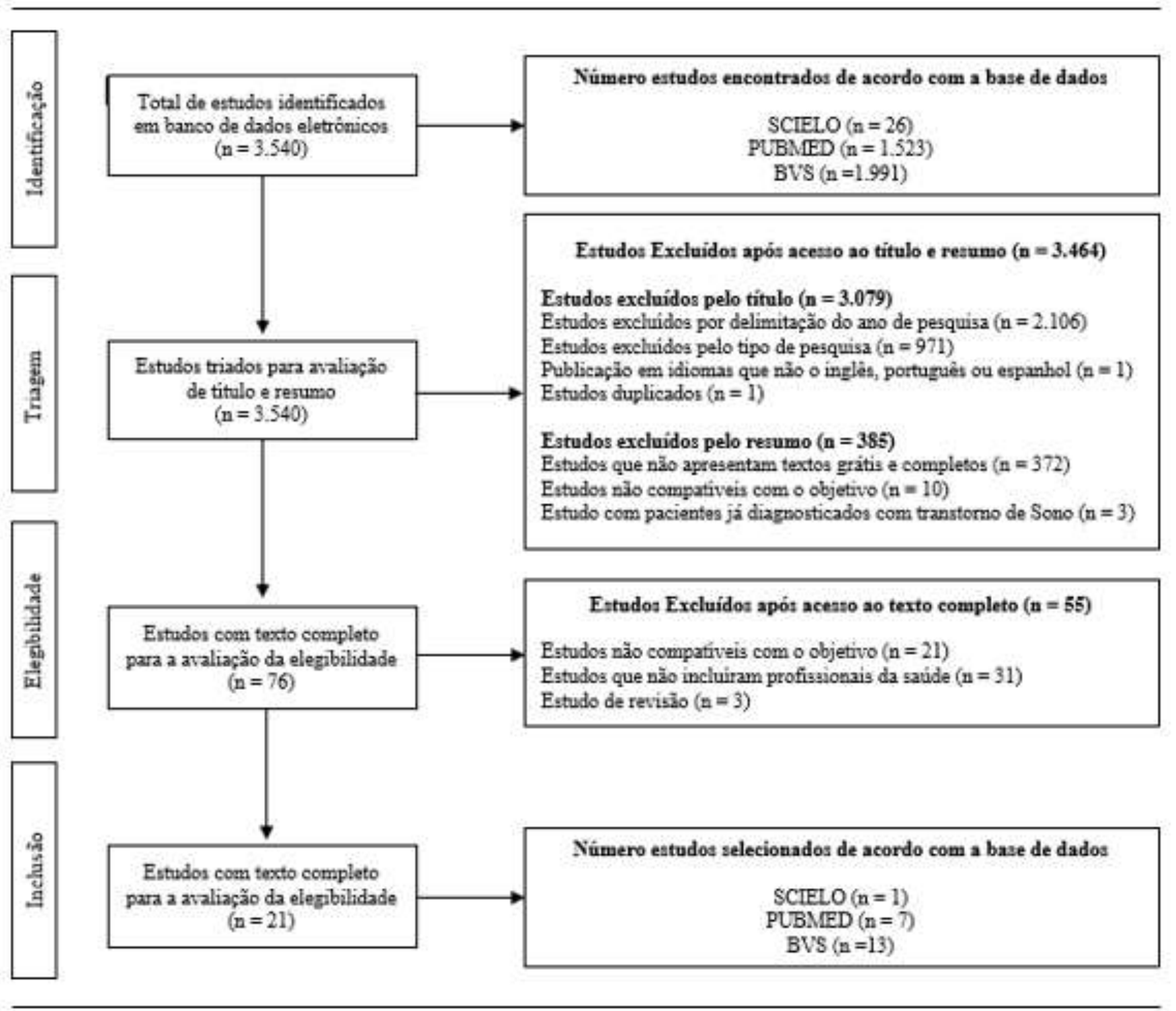

Fonte: Adaptado pelos autores do Preferred Reporting Items for Systematic Reviews and Meta-Analyses (PRISMA 2009).

Basner et al. (2017) relata que a fadiga causada pela perda de sono é um risco para a segurança do médico e do paciente, mas os dados objetivos sobre o sono e o estado de alerta do médico em diferentes horários de serviço são escassos. Deste modo, realizou um estudo que quantificou objetivamente as diferenças na duração do sono e alerta entre médicos internos que trabalham em turnos noturnos prolongados e residentes que não trabalham ou raramente trabalham em turnos noturnos prolongados.

Basner et al. (2019) selecionaram aleatoriamente 63 programas de residência em medicina interna nos Estados Unidos para seguir as políticas padrão de horário de trabalho de 2011 ou políticas flexíveis que mantinham uma semana de trabalho de 80 horas sem limites na duração do turno ou folga obrigatória entre os turnos. A duração do sono e a sonolência matinal e o estado de alerta foram comparados entre os dois grupos por meio de um desenho de não inferioridade, com medidas de desfecho incluindo a duração do sono medida com actigrafia, a Escala de Sonolência de Karolinska (com pontuações variando de 1 [extremamente alerta] a 9 [extremamente sonolento, lutando contra o sono]), e um breve teste computadorizado de vigilância psicomotora (PVT-B), com longos tempos de resposta (lapsos) indicando redução do estado de alerta.

Griepentrog et al. (2018) relatam que o trabalho em turnos pode perturbar a homeostase circadiana e resultar em fadiga, sonolência excessiva e redução da qualidade de vida. Foi demonstrado que a terapia da luz confere efeitos positivos em 
trabalhadores noturnos. Procuramos determinar se a exposição prolongada à luz forte durante o turno da noite reduz a sonolência e melhora o desempenho psicomotor entre enfermeiras de UTI. Um total de 43 pares combinados completaram ambas as exposições de iluminação e foram analisados. Quando expostos a iluminação de alta iluminância, os indivíduos experimentaram escores de sonolência reduzidos na Escala de Sonolência de Stanford do que quando expostos à iluminação hospitalar padrão, no entanto, eles cometeram mais erros psicomotores durante a pesquisa.

De acordo com Mizobe et al. (2019) estudos têm mostrado que a privação de sono pode reduzir a empatia entre estudantes de medicina. No entanto, pouco se sabe sobre a empatia após uma noite de plantão do tipo on call ou plantão noturno entre os médicos residentes. Assim, esse estudo objetivou verificar se o plantão do tipo on call ou o plantão noturno reduz a empatia dos médicos. Os pesquisadores utilizaram-se de uma pesquisa cruzada multicêntrica randomizada usando a Escala de Empatia Médica de Jefferson (JSE), submetendo um total de 260 médicos que trabalharam em hospitais acadêmicos e hospitais comunitários no Japão em 2016, randomizando-os em dois grupos e aplicando a escala antes e após os períodos noturnos de plantão.

Nishida et al. (2017) objetivou determinar a possibilidade de deterioração da função cerebral de residentes fatigados e privados de sono usando técnicas de neuroimagem, ao realizar procedimento de coleta de sangue de vasos artificiais instalados no braço de um cooperador normal, para a avaliação das possíveis variações durante o período dos plantões. O sangue foi coletado em um horário semelhante do dia, antes e depois do serviço noturno. Para avaliar as condições de sono durante o plantão noturno, os participantes utilizaram aparelhos de actigrafia durante todo o plantão noturno. Mudanças na hemodinâmica cerebral, durante o curso da coleta de sangue, foram medidas usando um sistema de topografia óptica vestível.

Tribis-Arrospe et al. (2020) basearam seu estudo na exploração da qualidade do sono e da adaptação ao turno do pessoal da ambulância de urgência e sua relação com fatores sociodemográficos, circadianos e laborais. Estudo transversal com 180 técnicos e enfermeiros (18-60 anos) da Rede de Transporte Urgente de Saúde do País Basco realizado por meio de inquéritos. O Índice de Qualidade do Sono de Pittsburgh (PSQI) foi usado para avaliar a qualidade do sono, a Escala de Adaptação de Turno de Trabalho (ATT), o Questionário de Tipo Circadiano (CTQ) de hábitos de sono e a Escala Composto de Maturidade (CSM) para avaliar o cronótipo.

Aydın Güçlü et al. (2019) chamam atenção para o aumento do risco de desenvolvimento de doenças crônicas, acidentes de trabalho, sintomas neurocomportamentais e diminuição da qualidade de vida devido a privação de sono. Diante disso, o estudo investigou o risco de apneia obstrutiva do sono em profissionais de saúde e os fatores associados a esse risco, aplicando o questionário de Berlim. Trabalhadores de saúde que concordaram em participar do estudo de diferentes centros foram incluídos no estudo, aplicando-se o questionário sobre as características demográficas dos participantes, histórico de tabagismo, comorbidades, plantão, número de crises convulsivas e duração do sono.

Leu et al. (2019) avaliou os efeitos da privação de sono em laparoscopia simulada por computador em 20 cirurgiões iniciantes após 24 horas de privação de sono e após repouso usando um treinador de realidade virtual. Os participantes foram designados aleatoriamente para realizar testes de simulador bem descansados ou privados de sono primeiro.

Liu et al. (2019) examinaram a prevalência de problemas de sono entre funcionários de hospitais terciários na China e identificaram os fatores associados. O estudo incluiu uma amostra representativa de 4.007 trabalhadores de saúde, incluindo médicos, técnicos médicos e funcionários de escritório, que foram selecionados usando um método de amostragem por conglomerados em vários estágios. No final das contas, 3.810 participantes completaram questionários válidos, gerando uma taxa de resposta de $95 \%$. Os participantes responderam a um questionário estruturado que coletou dados demográficos, problemas de sono, emprego, dor crônica e comportamentos relacionados à saúde. Um modelo de regressão logística de dois níveis foi construído para examinar os determinantes dos problemas de sono.

Abbott et al. (2019) avaliaram a relação entre medidas objetivas de tempo de sono-vigília e estabilidade com risco de 
doença cardiometabólica. Enquanto isso, Barger et al. (2017) destacaram o insuficiente conhecimento científico acerca da duração curta de sono, apnéia obstrutiva do sono, e o trabalho noturno em relação a associação com o risco de eventos cardiovasculares recorrentes em pacientes após uma síndrome coronária aguda.

Para Francis et al. (2019) a medicina de emergência, por sua natureza, requer trabalho por turnos que segue um padrão errático e imprevisível. Diante deste desafio, foi elaborada a hipótese no estudo que muitos médicos de emergência tomaram medidas para minimizar a própria privação do sono através do uso de soníferos farmacológicos. A extensão e natureza dos benefícios do uso de soníferos farmacológicos não é bem estudado. Por isso, os autores buscaram descrever o uso desses fármacos para a manutenção do sono entre médicos de emergência em um ambiente de cuidados terciários canadense.

Em relação a qualidade do cuidado Arzalier-Daret et al. (2018) apontaram que a privação de sono tem sido associada a um aumento na incidência de erros médicos e pode colocar em risco a segurança dos pacientes durante o gerenciamento de crises médicas. Testaram essa hipótese a partir da avaliação do efeito da privação de sono no manejo de crises simuladas de anestesia por residentes de anestesiologia. Além disso, de forma semelhante, Gómez-García et al. (2016) almejaram determinar a relação entre as características dos ambientes de trabalho dos enfermeiros em hospitais do Sistema Único de Saúde (SNHS) espanhol com a qualidade dos cuidados relatados pelos enfermeiros e como os cuidados eram prestados através de diferentes esquemas de turnos, além de examinar a relação entre satisfação no trabalho, burnout, qualidade do sono e sonolência diurna de enfermeiras e trabalho por turnos.

Patterson et al. (2017) dizem que a maioria dos médicos dos Serviços de Emergência Médica (EMS) que trabalham em turnos de longa duração e mais de 50\% relatam sono inadequado, má qualidade do sono e/ou recuperação insuficiente entre os turnos. O estudo piloto SleepTrackTXT (ClinicalTrials.gov, NCT02063737) mostrou que o uso do telefone celular mensagens de texto poderia impactar na fadiga e na sonolência EMS durante turnos de longa duração. Com isso, esta nova versão do ensaio objetivou que o SleepTrackTXT2 alavancasse as lições aprendidas com o primeiro estudo SleepTrackTXT, além de testar uma intervenção aprimorada direcionada aos médicos de SEM da aeromedicina.

Guerra et al. (2016) avaliou o sono, a qualidade de vida e o humor de profissionais de enfermagem de unidades de terapia intensiva pediátrica, os quais foram agrupados nos turnos matutino, vespertino e noturno por meio de instrumentos como: Morningness-Eveningness Questionnaire; Índice de Qualidade do Sono de Pittsburgh; Escala de Sonolência de Epworth; Questionário genérico de avaliação de qualidade de vida (SF-36); Inventário de Depressão de Beck; Inventário de ansiedade de Beck; Estado-Trait Anxiety Inventory.

Haile et al. (2019) avaliou a prevalência e os fatores associados do distúrbio do sono durante o trabalho em turnos entre enfermeiras que trabalham em hospitais do governo federal da Etiópia em Addis Abeba. Para isso adotou um estudo transversal usando questionários estruturados como a Classificação Internacional de Distúrbios do Sono - Terceira Edição, a Escala de Insônia de Bargen e/ou Escala de Sonolência de Epworth para medir o desfecho distúrbio do sono no trabalho por turnos. 
Figura 2. Temas mais representativos em cada classe.

\begin{tabular}{|c|c|c|c|c|c|}
\hline Autor/ Ano & Título & $\begin{array}{l}\text { Biblioteca } \\
\text { Virtual }\end{array}$ & Objetivo & Método & $\begin{array}{l}\text { Níveis de } \\
\text { Evidência }\end{array}$ \\
\hline $\begin{array}{l}\text { Barger, et al. } \\
\text { (2019) }\end{array}$ & $\begin{array}{l}\text { Effects on resident work } \\
\text { hours, sleep duration, and } \\
\text { work experience in a } \\
\text { randomized order safety } \\
\text { trial evaluating resident- } \\
\text { physician schedules. }\end{array}$ & BVS & $\begin{array}{l}\text { Comparar as horas de trabalho do } \\
\text { médico residente e o sono. }\end{array}$ & $\begin{array}{l}\text { Ensaio clínico } \\
\text { multicêntrico } \\
\text { cruzado } \\
\text { randomizado. }\end{array}$ & Nível 2 \\
\hline $\begin{array}{l}\text { Blackwell, et } \\
\text { al. (2019) }\end{array}$ & $\begin{array}{l}\text { Design and recruitment of } \\
\text { the randomized order safety } \\
\text { trial evaluating resident- } \\
\text { physician schedules. }\end{array}$ & BVS & $\begin{array}{l}\text { Avaliar a eficácia da eliminação de } \\
\text { turnos tradicionais de } 24 \text { horas ou mais } \\
\text { para o segundo ano ou mais médicos } \\
\text { residentes em pediatria intensiva } \\
\text { unidades de atendimento. }\end{array}$ & $\begin{array}{l}\text { Estudo } \\
\text { multicêntrico } \\
\text { não cego. }\end{array}$ & Nível 3 \\
\hline $\begin{array}{l}\text { Basner, et al. } \\
\quad(2017)\end{array}$ & $\begin{array}{l}\text { Sleep and Alertness in } \\
\text { Medical Interns and } \\
\text { Residents: } \\
\text { Observational Study on the } \\
\text { Role of Extended Shifts. }\end{array}$ & BVS & $\begin{array}{l}\text { Este estudo quantificou objetivamente } \\
\text { as diferenças na duração do sono e } \\
\text { alerta entre médicos internos que } \\
\text { trabalham em turnos noturnos } \\
\text { prolongados e residentes que não } \\
\text { trabalham ou raramente trabalham em } \\
\text { turnos noturnos prolongados. }\end{array}$ & $\begin{array}{l}\text { Ensaios clínicos } \\
\text { controlados } \\
\text { randomizados. }\end{array}$ & Nível 2 \\
\hline $\begin{array}{l}\text { Basner, et al. } \\
\quad(2019)\end{array}$ & $\begin{array}{l}\text { Sleep and Alertness in a } \\
\text { Duty-Hour Flexibility Trial } \\
\text { in Internal Medicine. }\end{array}$ & BVS & $\begin{array}{l}\text { Estabelecer se o sono e o estado de } \\
\text { alerta entre os estagiários em } \\
\text { programas flexíveis não eram inferiores } \\
\text { aos dos estagiários em programas } \\
\text { padrão, de acordo com as margens de } \\
\text { não inferioridade pré-especificadas. }\end{array}$ & $\begin{array}{l}\text { Ensaio clínico } \\
\text { randomizado. }\end{array}$ & Nível 2 \\
\hline $\begin{array}{l}\text { Griepentrog, } \\
\text { et al. }(\mathbf{2 0 1 8})\end{array}$ & $\begin{array}{l}\text { Bright environmental light } \\
\text { improves the sleepiness of } \\
\text { nightshift ICU nurses. }\end{array}$ & BVS & $\begin{array}{l}\text { Determinar se a exposição prolongada } \\
\text { à luz forte durante o turno da noite } \\
\text { reduz a sonolência e melhora o } \\
\text { desempenho psicomotor entre } \\
\text { enfermeiras de UTI. }\end{array}$ & $\begin{array}{l}\text { Ensaio clínico } \\
\text { cruzado, } \\
\text { randomizado e } \\
\text { unicêntrico. }\end{array}$ & Nível 3 \\
\hline $\begin{array}{l}\text { Mizobe, et } \\
\text { al. (2019) }\end{array}$ & $\begin{array}{l}\text { A night on call or an } \\
\text { overnight shift does not } \\
\text { reduce residents' empathy: a } \\
\text { randomized crossover } \\
\text { multicenter survey. }\end{array}$ & BVS & $\begin{array}{l}\text { Verificar se o plantão noturno ou o } \\
\text { plantão noturno reduz a empatia dos } \\
\text { médicos. }\end{array}$ & $\begin{array}{l}\text { Pesquisa cruzada } \\
\text { multicêntrica } \\
\text { randomizada. }\end{array}$ & Nível 2 \\
\hline $\begin{array}{l}\text { Nishida, et } \\
\text { al. (2017) }\end{array}$ & $\begin{array}{l}\text { Night duty and decreased } \\
\text { brain activity of medical } \\
\text { residents: a wearable optical } \\
\text { topography study. }\end{array}$ & BVS & $\begin{array}{l}\text { Determinar a possibilidade de } \\
\text { deterioração da função cerebral de } \\
\text { residentes fatigados e privados de sono } \\
\text { usando técnicas de neuroimagem. }\end{array}$ & $\begin{array}{l}\text { Pesquisa cruzada } \\
\text { unicêntrica } \\
\text { randomizada. }\end{array}$ & Nível 2 \\
\hline $\begin{array}{l}\text { Neufeld, et } \\
\text { al. (2017) }\end{array}$ & $\begin{array}{l}\text { Exploratory Study of Heart } \\
\text { Rate Variability and Sleep } \\
\text { among Emergency Medical } \\
\text { Services Shift Workers. }\end{array}$ & BVS & $\begin{array}{l}\text { Caracterizar a continuidade e a duração } \\
\text { do sono e medir o equilíbrio } \\
\text { autonômico cardíaco noturno por meio } \\
\text { da variabilidade da frequência cardíaca } \\
\text { em um grupo de técnicos de } \\
\text { emergência médica de plantão e } \\
\text { desligamento. }\end{array}$ & $\begin{array}{l}\text { Pesquisa } \\
\text { quantitativa, } \\
\text { prospectiva, } \\
\text { descritiva e } \\
\text { analítica. }\end{array}$ & Nível 3 \\
\hline $\begin{array}{l}\text { Tribis- } \\
\text { Arrospe, et } \\
\text { al. }(2020)\end{array}$ & $\begin{array}{l}\text { Calidad del sueño y } \\
\text { adaptación a los turnos } \\
\text { rotatorios en trabajadores de } \\
\text { ambulancias de emergencias } \\
\text { del País Vasco. }\end{array}$ & SCIELO & $\begin{array}{l}\text { Explorar a qualidade do sono e a } \\
\text { adaptação ao turno do pessoal da } \\
\text { ambulância de urgência e sua relação } \\
\text { com fatores sociodemográficos, } \\
\text { circadianos e laborais. }\end{array}$ & $\begin{array}{l}\text { Estudo } \\
\text { transversal, } \\
\text { quantitativo, } \\
\text { descritivo. }\end{array}$ & Nível 4 \\
\hline $\begin{array}{l}\text { Aydın } \\
\text { Güçlï, et al. } \\
(\mathbf{2 0 1 9})\end{array}$ & $\begin{array}{l}\text { Assessment of the risk of } \\
\text { obstructive sleep apnoea } \\
\text { syndrome among healthcare } \\
\text { workers. }\end{array}$ & PubMed & $\begin{array}{l}\text { O objetivo do nosso estudo é avaliar a } \\
\text { prevalência de ronco e risco de apneia } \\
\text { obstrutiva do sono entre profissionais } \\
\text { de saúde (PS) por meio do Questionário } \\
\text { de Berlim. }\end{array}$ & $\begin{array}{l}\text { Estudo analítico } \\
\text { transversal e } \\
\text { quantitativo. }\end{array}$ & Nível 3 \\
\hline $\begin{array}{l}\text { Leu, et al. } \\
\text { (2019) }\end{array}$ & $\begin{array}{l}\text { Impact of Sleep Deprivation } \\
\text { on Surgical Laparoscopic } \\
\text { Performance in Novices: A } \\
\text { Computer-based Crossover } \\
\text { Study. }\end{array}$ & PubMed & $\begin{array}{l}\text { Avaliar a atividade motora dos novatos } \\
\text { em cirurgia mediante a privação de } \\
\text { sono. }\end{array}$ & $\begin{array}{l}\text { Estudo } \\
\text { quantitativo, } \\
\text { analítico } \\
\text { descritivo, } \\
\text { randomizado. }\end{array}$ & Nível 3 \\
\hline $\begin{array}{l}\text { Liu, et al. } \\
\text { (2019) }\end{array}$ & $\begin{array}{lrr}\text { Sleep } & \text { problems } & \text { of } \\
\text { healthcare } & \text { workers } & \text { in }\end{array}$ & BVS & $\begin{array}{l}\text { Examinar a prevalência de problemas } \\
\text { de sono entre funcionários de hospitais }\end{array}$ & $\begin{array}{c}\text { Estudo } \\
\text { multicêntrico, }\end{array}$ & Nível 3 \\
\hline
\end{tabular}




\begin{tabular}{|c|c|c|c|c|c|}
\hline & $\begin{array}{l}\text { tertiary hospital and } \\
\text { influencing factors } \\
\text { identified through a } \\
\text { multilevel analysis: a cross- } \\
\text { sectional study in China. }\end{array}$ & & $\begin{array}{l}\text { terciários na China e identificar os } \\
\text { fatores associados. }\end{array}$ & $\begin{array}{l}\text { transversal, } \\
\text { quantitativo e } \\
\text { descritivo. }\end{array}$ & \\
\hline $\begin{array}{l}\text { Abbott, et al. } \\
\text { (2019) }\end{array}$ & $\begin{array}{l}\text { Sleep Timing, Stability, and } \\
\text { BP in the Sueño Ancillary } \\
\text { Study of the Hispanic } \\
\text { Community Health } \\
\text { Study/Study of Latinos. }\end{array}$ & BVS & $\begin{array}{l}\text { O objetivo deste estudo foi avaliar a } \\
\text { relação entre medidas objetivas de } \\
\text { tempo de sono-vigília e estabilidade } \\
\text { com risco de doença cardiometabólica. }\end{array}$ & $\begin{array}{c}\text { Estudo } \\
\text { multicêntrico, } \\
\text { transversal e de } \\
\text { base } \\
\text { populacional. }\end{array}$ & Nível 3 \\
\hline $\begin{array}{l}\text { Barger, et al. } \\
\quad \text { (2017) }\end{array}$ & $\begin{array}{l}\text { Short Sleep Duration, } \\
\text { Obstructive Sleep Apnea, } \\
\text { Shiftwork, and the Risk of } \\
\text { Adverse Cardiovascular } \\
\text { Events in Patients After an } \\
\text { Acute Coronary Syndrome. }\end{array}$ & BVS & $\begin{array}{l}\text { O estudo levanta a hipótese que } \\
\text { prospectivamente haveria uma } \\
\text { associação entre curta duração do sono, } \\
\text { OSA, trabalho noturno em turnos e } \\
\text { eventos cardiovasculares em uma } \\
\text { grande população de pacientes com } \\
\text { SCA. }\end{array}$ & $\begin{array}{l}\text { Ensaio clínico } \\
\text { randomizado } \\
\text { duplo-cego. }\end{array}$ & Nível 2 \\
\hline $\begin{array}{l}\text { Francis, et } \\
\text { al. (2019) }\end{array}$ & $\begin{array}{l}\text { Use of Pharmacologic Sleep } \\
\text { Aids and Stimulants Among } \\
\text { Emergency Medicine Staff } \\
\text { Physicians in a Canadian } \\
\text { Tertiary Care Setting: A } \\
\text { Web-Based Survey. }\end{array}$ & PubMed & $\begin{array}{l}\text { Descrever o uso de soníferos } \\
\text { farmacológicos entre médicos de } \\
\text { emergência em um ambiente de } \\
\text { cuidados terciários canadenses. }\end{array}$ & $\begin{array}{l}\text { Pesquisa } \\
\text { descritiva } \\
\text { transversal e } \\
\text { quantitativa. }\end{array}$ & Nível 3 \\
\hline $\begin{array}{l}\text { Arzalier- } \\
\text { Daret, et al. } \\
(2018)\end{array}$ & $\begin{array}{l}\text { Effect of sleep deprivation } \\
\text { after a night shift duty on } \\
\text { simulated crisis } \\
\text { management by residents in } \\
\text { anaesthesia. A randomised } \\
\text { crossover study. }\end{array}$ & PubMed & $\begin{array}{l}\text { Avaliar o efeito da privação de sono no } \\
\text { manejo de crises simuladas de anestesia } \\
\text { por residentes de anestesiologia. }\end{array}$ & $\begin{array}{l}\text { Estudo } \\
\text { randomizado, } \\
\text { comparativo e } \\
\text { cruzado } \\
\text { unicêntrico. }\end{array}$ & Nível 2 \\
\hline $\begin{array}{l}\text { Gómez- } \\
\text { García, et al. } \\
(\mathbf{2 0 1 6 )}\end{array}$ & $\begin{array}{l}\text { Nurses' sleep quality, work } \\
\text { environment and quality of } \\
\text { care in the Spanish National } \\
\text { Health System: } \\
\text { observational study among } \\
\text { different shifts }\end{array}$ & BVS & $\begin{array}{l}\text { Determinar a relação entre as } \\
\text { características dos ambientes de } \\
\text { trabalho dos enfermeiros em hospitais } \\
\text { do Sistema Único de Saúde (SNHS) } \\
\text { espanhol com a qualidade dos cuidados } \\
\text { relatados pelos enfermeiros e como os } \\
\text { cuidados eram prestados através de } \\
\text { diferentes esquemas de turnos. }\end{array}$ & $\begin{array}{l}\text { Estudo } \\
\text { multicêntrico, } \\
\text { observacional, } \\
\text { descritivo, } \\
\text { transversal. }\end{array}$ & Nível 3 \\
\hline $\begin{array}{l}\text { Patterson, et } \\
\text { al. (2017) }\end{array}$ & $\begin{array}{l}\text { Fatigue mitigation with } \\
\text { SleepTrackTXT2 in air } \\
\text { medical emergency care } \\
\text { systems: study protocol for a } \\
\text { randomized controlled trial. }\end{array}$ & BVS & $\begin{array}{l}\text { Alavancar as lições aprendidas com o } \\
\text { primeiro estudo SleepTrackTXT e } \\
\text { testar uma intervenção aprimorada } \\
\text { direcionada aos médicos de EMS da } \\
\text { aeromedicina. }\end{array}$ & $\begin{array}{l}\text { Ensaio clínico } \\
\text { randomizado } \\
\text { multicêntrico. }\end{array}$ & Nível 2 \\
\hline $\begin{array}{l}\text { Guerra, et } \\
\text { al. (2016) }\end{array}$ & $\begin{array}{l}\text { Sleep, quality of life and } \\
\text { mood of nursing } \\
\text { professionals of pediatric } \\
\text { intensive care units }\end{array}$ & PubMed & $\begin{array}{l}\text { Avaliar sono, qualidade de vida e } \\
\text { humor em profissionais de enfermagem } \\
\text { em Unidades de Terapia Intensiva } \\
\text { Infantil. }\end{array}$ & $\begin{array}{c}\text { Estudo } \\
\text { quantitativo, } \\
\text { transversal e } \\
\text { descritivo. } \\
\end{array}$ & Nível 4 \\
\hline $\begin{array}{l}\text { Haile, et al. } \\
\text { (2019) }\end{array}$ & $\begin{array}{l}\text { Shift work sleep disorders } \\
\text { and associated factors } \\
\text { among nurses at federal } \\
\text { government hospitals in } \\
\text { Ethiopia: a cross-sectional } \\
\text { study }\end{array}$ & PubMed & $\begin{array}{l}\text { Avaliar a prevalência e os fatores } \\
\text { associados ao distúrbio do sono durante } \\
\text { o trabalho em turnos entre enfermeiras } \\
\text { que trabalham em hospitais do governo } \\
\text { federal da Etiópia em Addis Abeba. }\end{array}$ & $\begin{array}{l}\text { Estudo } \\
\text { transversal, } \\
\text { quantitativo e } \\
\text { descritivo. }\end{array}$ & Nível 4 \\
\hline $\begin{array}{l}\text { Dutheil, et } \\
\text { al. (2017) }\end{array}$ & $\begin{array}{l}\text { Maximal tachycardia and } \\
\text { high cardiac strain during } \\
\text { night shifts of emergency } \\
\text { physicians }\end{array}$ & PubMed & $\begin{array}{l}\text { Comparar a taquicardia e o esforço } \\
\text { cardíaco entre turnos de } 24 \text { horas e } \\
\text { turnos noturnos de } 14 \text { horas em } \\
\text { médicos de emergência e investigar os } \\
\text { principais fatores que influenciam a } \\
\text { taquicardia e o esforço cardíaco. }\end{array}$ & $\begin{array}{l}\text { Ensaio clínico } \\
\text { randomizado. }\end{array}$ & Nível 2 \\
\hline
\end{tabular}

Fonte: Autores.

\section{Discussão}

O presente estudo apontou que, de maneira geral, a qualidade do sono está diretamente ligada a sua extensa jornada de trabalho. Entre os vinte e dois artigos utilizados, todos em suas partes corroboram para a hipótese de que profissionais em 
melhores condições de trabalho, com horários previamente planejados e escalonados e sem sistema rotativo por turnos no plantão noturno, aceitam e respondem com mais aceitação (Francis et al., 2019). Ainda assim, é preciso notar que as relações de trabalho as quais o profissional está ligado, seus espaços e a qualidade deste está diretamente ligado à sua resposta de qualidade do sono (Leu et al., 2019).

Assim, as evidências apresentadas demonstraram que trabalhadores que exercem suas atividades laborais em sistema de organização noturno, apresentam frequentemente alterações no sono, o que acarretam diversas outras consequências negativas, interferindo não só na qualidade do sono, mas também na qualidade de vida e da saúde dos plantonistas. Associado a isso, observa-se manifestações como quadros de depressão, irritabilidade, diminuição da autoestima e labilidade de humor nos trabalhadores (Guerra et al., 2016).

Um outro estudo, evidenciou, uma má qualidade do sono e do ciclo sono-vigília; com maior prevalência do período de despertar e adormecer durante os dias de semana e fins de semana estatisticamente diferentes, sugerindo que esses profissionais não têm o tempo de descanso necessário durante a semana. Verificou-se também a presença de alterações físicas e mentais associadas ao trabalho em turno, independentemente do turno trabalhado, e uma pior qualidade do sono nos profissionais do turno noturno (Guerra et al., 2016).

Dessa forma, trabalhadores da saúde em regime de plantão, apresentam alto risco para apneia do sono. Idade, sexo, altura, peso, IMC e hipertensão estão associados a esse maior risco. Nos estudos abordados, mais da metade destes trabalhadores noturnos apresentam o fator obesidade prevalente, relacionado a Síndrome da apneia obstrutiva do sono, o que aumenta consideravelmente os riscos para outros eventos, principalmente cardiovasculares (Aydın Güçlü et al., 2019).

Em contrapartida, foi encontrada uma relação direta entre idade, sexo e risco de apneia do sono, o que indicou que esse risco aumenta com o envelhecimento. Assim, o sono desordenado é um importante problema de saúde pública, que reduz a qualidade de vida ao afetar negativamente a produtividade e o desempenho, com horários de plantões estendidos, aumentando o risco de distúrbios do sono (Leu et al., 2019).

Os turnos rotativos são frequentemente associados a períodos de sono mais curtos do que o normal e interrompidos em uma fase circadiana adversa. Turnos rotativos, incluindo turnos noturnos, podem causar alterações na secreção de hormônios, visto que o trabalho por turnos tem uma correlação significativa com os níveis séricos do hormônio tireoidiano e prolactina. Assim, a longa jornada noturna de trabalho e a consequente interferência nos ritmos circadianos estão associados à ocorrência de transtorno do trabalho em turnos entre os profissionais em regime de rápida rotação (Haile et al. 2019).

Pode-se afirmar que o excesso de atividades e altas jornadas de trabalho, podem contribuir diretamente no surgimento de situações e/ou problemáticas que afetem a vida social, psicológica e fisiológica dos trabalhadores da saúde em regime de plantão noturno, afetando negativamente não só os profissionais, mas também o ambiente de trabalho, a equipe e os próprios pacientes, partindo-se do princípio que um profissional emocionalmente desgastado pode não realizar satisfatoriamente um bom atendimento (Neufeld et al., 2017).

Portanto, a partir dos resultados evidenciados no trabalho de pesquisa científica, é pertinente destacar a importância de novas medidas de enfrentamento para minimizar os problemas no ambiente de trabalho e melhorar a vida dos trabalhadores e a qualidade da assistência prestadas a estes, de modo que haja mais pesquisas e implementação de programas que possam diminuir os fatores estressores e melhor adequar as escalas de trabalho.

\section{Considerações Finais}

Os profissionais que mais sofrem conflitos diários em suas jornadas de trabalho são os que os realizam elas em regime de plantão hospitalar noturno, demonstrando que em geral há uma baixa qualidade de sono dos profissionais que influenciam nas escolhas profissionais e mesmo pessoais desses indivíduos. Desse modo, protocolos de cuidado e vigilância em saúde para 
estes profissionais são de extrema importância, objetivando sanar ou reduzir problemas como apneia do sono, conflitos familiares, ansiedade, depressão, problemas cardiovasculares e erros médicos. Assim, também é possível afirmar que mesmo com o trabalho hospitalar em regime de plantão noturno, alguns profissionais podem se beneficiar utilizando escalas de trabalho mais adequadas e divididas em equipes maiores, dispondo de horários bem compartilhados durante o regime de plantão noturno.

\section{Referências}

Abbott, S. M., Weng, J., Reid, K. J., Daviglus, M. L., Gallo, L. C., Loredo, J. S., Nyenhuis, S. M., Ramos, A. R., Shah, N. A., Sotres-Alvarez, D., Patel, S. R., \& Zee, P. C. (2019). Sleep Timing, Stability, and BP in the Sueño Ancillary Study of the Hispanic Community Health Study/Study of Latinos. Chest, 155(1), 60-68. https://doi.org/10.1016/j.chest.2018.09.018

Arzalier-Daret, S., Buléon, C., Bocca, M. L., Denise, P., Gérard, J. L., \& Hanouz, J. L. (2018). Effect of sleep deprivation after a night shift duty on simulated crisis management by residents in anaesthesia. A randomised crossover study. Anaesthesia, critical care \& pain medicine, 37(2), 161-166. https://doi.org/10.1016/j.accpm.2017.05.010

Aydın Güçlü, Ö., Karadağ, M., İntepe, Y. S., Acıcan, T., Arslan, S., \& Sertoğullarından, B. (2019). Assessment of the risk of obstructive sleep apnoea syndrome among healthcare workers. Sağlık çalışanlarında obstrüktif uyku apne sendromu riskinin değerlendirilmesi. Tuberkuloz ve toraks, 67(1), 47-54. https://doi.org/10.5578/tt.68166

Barger, L. K., Rajaratnam, S., Cannon, C. P., Lukas, M. A., Im, K., Goodrich, E. L., Czeisler, C. A., \& O'Donoghue, M. L. (2017). Short Sleep Duration, Obstructive Sleep Apnea, Shiftwork, and the Risk of Adverse Cardiovascular Events in Patients After an Acute Coronary Syndrome. Journal of the American Heart Association, 6(10), e006959. https://doi.org/10.1161/JAHA.117.006959

Barger, L. K., Sullivan, J. P., Blackwell, T., O'Brien, C. S., St Hilaire, M. A., Rahman, S. A., Phillips, A., Qadri, S., Wright, K. P., Segar, J. L., McGuire, J. K., Vitiello, M. V., de la Iglesia, H. O., Poynter, S. E., Yu, P. L., Zee, P., Sanderson, A. L., Halbower, A. C., Lockley, S. W., Landrigan, C. P., Stone, K.L., Czeisler, C.A., ROSTERS Study Group (2019). Effects on resident work hours, sleep duration, and work experience in a randomized order safety trial evaluating residentphysician schedules (ROSTERS). Sleep, 42(8), zsz110. https://doi.org/10.1093/sleep/zsz110

Basner, M., Asch, D. A., Shea, J. A., Bellini, L. M., Carlin, M., Ecker, A. J., Malone, S. K., Desai, S. V., Sternberg, A. L., Tonascia, J., Shade, D. M., Katz, J. T., Bates, D. W., Even-Shoshan, O., Silber, J. H., Small, D. S., Volpp, K. G., Mott, C. G., Coats, S., Mollicone, D. J., Dinges, D. F., iCOMPARE Research Group (2019). Sleep and Alertness in a Duty-Hour Flexibility Trial in Internal Medicine. The New England journal of medicine, 380(10), 915-923. https://doi.org/10.1056/NEJMoa1810641

Basner, M., Dinges, D. F., Shea, J. A., Small, D. S., Zhu, J., Norton, L., Ecker, A. J., Novak, C., Bellini, L. M., \& Volpp, K. G. (2017). Sleep and Alertness in Medical Interns and Residents: An Observational Study on the Role of Extended Shifts. Sleep, 40(4), zsx027. https://doi.org/10.1093/sleep/zsx027

Blackwell, T., Kriesel, D. R., Vittinghoff, E., O'Brien, C. S., Sullivan, J. P., Viyaran, N. C., Rahman, S. A., Lockley, S. W., Barger, L. K., Halbower, A. C., Poynter, S. E., Wright, K. P., Jr, Yu, P. L., Zee, P. C., Landrigan, C. P., Czeisler, C. A., Stone, K. L., \& ROSTERS Study Group (2019). Design and recruitment of the randomized order safety trial evaluating resident-physician schedules (ROSTERS) study. Contemporary clinical trials, 80, 22-33. https://doi.org/10.1016/j.cct.2019.03.005

Dutheil, F., Marhar, F., Boudet, G., Perrier, C., Naughton, G., Chamoux, A., Huguet, P., Mermillod, M., Saâdaoui, F., Moustafa, F., \& Schmidt, J. (2017). Maximal tachycardia and high cardiac strain during night shifts of emergency physicians. International archives of occupational and environmental health, 90(6), 467-480. https://doi.org/10.1007/s00420-017-1211-5

Francis, M. N., Wishart, I. M., Williamson, T., \& Iverach, R. (2019). Use of Pharmacologic Sleep Aids and Stimulants Among Emergency Medicine Staff Physicians in a Canadian Tertiary Care Setting: A Web-Based Survey. Annals of emergency medicine, 73(4), 325-329.

Gómez-García, T., Ruzafa-Martínez, M., Fuentelsaz-Gallego, C., Madrid, J. A., Rol, M. A., Martínez-Madrid, M. J., Moreno-Casbas, T., \& SYCE and RETICEF Group (2016). Nurses' sleep quality, work environment and quality of care in the Spanish National Health System: observational study among different shifts. BMJ open, 6(8), e012073. https://doi.org/10.1136/bmjopen-2016-012073

Griepentrog, J. E., Labiner, H. E., Gunn, S. R., \& Rosengart, M. R. (2018). Bright environmental light improves the sleepiness of nightshift ICU nurses. Critical care (London, England), 22(1), 295. https://doi.org/10.1186/s13054-018-2233-4

Guerra, P.C., Oliveira, N.F., Terreri, M.T., Len, C.A. (2016). Sleep, quality of life and mood of nursing professionals of pediatric intensive care units. Revista da Escola de Enfermagem da USP, 50(2): 279-85. 10.1590/S0080- 623420160000200014.

Haile, K. K., Asnakew, S., Waja, T., \& Kerbih, H. B. (2019). Shift work sleep disorders and associated factors among nurses at federal government hospitals in Ethiopia: a cross-sectional study. BMJ open, 9(8), e029802. https://doi.org/10.1136/bmjopen-2019-029802

Leu, S., Staerkle, R. F., Gaukel, S., Fink, L., Soll, C., Aasen, D. M., Liechty, K., Vitz, M., Ramseier, L. E., \& Vuille-Dit-Bille, R. N. (2019). Impact of Sleep Deprivation on Surgical Laparoscopic Performance in Novices: A Computer-based Crossover Study. Surgical laparoscopy, endoscopy \& percutaneous techniques, 29(3), 162-168. https://doi.org/10.1097/SLE.0000000000000657

Liu, H., Liu, J., Chen, M., Tan, X., Zheng, T., Kang, Z., Gao, L., Jiao, M., Ning, N., Liang, L., Wu, Q., \& Hao, Y. (2019). Sleep problems of healthcare workers in tertiary hospital and influencing factors identified through a multilevel analysis: a cross-sectional study in China. BMJ open, 9(12), e032239. https://doi.org/10.1136/bmjopen-2019-032239

Mizobe, M., Kataoka, H., Yamagami, H., Ito, C., Koyama, Y., Yawata, E., \& Shiga, T. (2019). A night on call or an overnight shift does not reduce residents' empathy: a randomized crossover multicenter survey. BMC medical education, 19(1), 391. https://doi.org/10.1186/s12909-019-1822-5 
Research, Society and Development, v. 10, n. 14, e351101421504, 2021

(CC BY 4.0) | ISSN 2525-3409 | DOI: http://dx.doi.org/10.33448/rsd-v10i14.21504

Neufeld, E. V., Carney, J. J., Dolezal, B. A., Boland, D. M., \& Cooper, C. B. (2017). Exploratory Study of Heart Rate Variability and Sleep among Emergency Medical Services Shift Workers. Prehospital emergency care : official journal of the National Association of EMS Physicians and the National Association of State EMS Directors, 21(1), 18-23. https://doi.org/10.1080/10903127.2016.1194928

Nishida, M., Kikuchi, S., Miwakeichi, F., \& Suda, S. (2017). Night duty and decreased brain activity of medical residents: a wearable optical topography study. Medical education online, 22(1), 1379345. https://doi.org/10.1080/10872981.2017.1379345

Patterson, P. D., Moore, C. G., Guyette, F. X., Doman, J. M., Sequeira, D., Werman, H. A., Swanson, D., Hostler, D., Lynch, J., Russo, L., Hines, L., Swecker, K., Runyon, M. S., \& Buysse, D. J. (2017). Fatigue mitigation with SleepTrackTXT2 in air medical emergency care systems: study protocol for a randomized controlled trial. Trials, 18(1), 254. https://doi.org/10.1186/s13063-017-1999-z

Phoi, Y. Y., \& Keogh, J. B. (2019). Dietary Interventions for Night Shift Workers: A Literature Review. Nutrients, 11(10), 2276. https://doi.org/10.3390/nu11102276

Tribis-Arrospe, B, Ballesteros-Peña, S, Abecia Inchaurregui, LC, Egea-Santaolalla, C, Guerra-Martin, L, \& Álvarez Ruiz de Larrinaga, A. (2020). Calidad del sueño y adaptación a los turnos rotatorios en trabajadores de ambulancias de emergencias del País Vasco. Anales del Sistema Sanitario de Navarra, 43(2), 189202. https://dx.doi.org/10.23938/assn.0871 